\title{
Considerations on the automation of a natural gas compressor station
}

\author{
Alina Maria Gligor* \\ "Lucian Blaga" University of Sibiu, Romania, Department of Industrial Engineering and Management, Emil Cioran str., 4, Sibiu, \\ Romania
}

\begin{abstract}
Natural gas compressor stations are very important components of a natural gas life cycle. Unfortunately, in Romania most of the existing natural gas compressor stations are equipped only with oldtype automation systems, with a high degree of physical and moral wear. The current paper describes the main elements and functions of an automation system that was implemented in a natural gas compressor station, offering an improved pressure control function, but also the possibility to control the compressors from a distance, for example from a central dispatching station.
\end{abstract}

\section{Introduction}

In many parts of the world and especially in the central part of Romania, where there are important reserves, natural gas represents an essential source of energy. However, being a fossil fuel with finite supply, it is of great importance to efficientise all aspects of its extraction, processing, transportation, storage, distribution and usage.

Natural gas compressor stations are processing units of the natural gas provided by the extraction wells, having the task to raise the gas pressure in order to allow either its transportation over large distances through main pipelines or the underground storage of the transported gases.

The main components of a natural gas compressor station are: the installation for separating and filtering gases; the technological piping system consisting of aspiration and evacuation collector pipes, bypasses, interconnections, gas stacks, evacuators; the installation for measuring basic technological parameters (such as flows, pressures, temperatures); compressor units; cooling systems for the compressed gases, oil circuits, compressor cylinders and engines; installations for separating the oil used for lubricating the compressor cylinders; the electric energy system; compressor station rooms; systems for detecting the presence of hazardous atmospheres; fire prevention systems, systems for isolating and depressurising in the event of an accident, video surveillance systems [1].

In natural gas compressor stations, the gas pressure can be increased by means of specific compressor units either turbocompressors, motocompressors or electrocompressors [2].

The compressor units in operation in Romania are mostly still fitted with older types of automation systems that display a high degree of physical and moral wear and most importantly lack the possibilities to transmit data over longer distances and to process numerically the data related to the compression process.
The current labour legislation and regulations demand better working conditions for operators in potentially hazardous environments, but also enhanced possibilities for the direct or remote control of equipments by operators and a minimal reaction time $[3,4]$.

Therefore, in the current paper, the author presents the implementation of a solution for a modern automation system for a natural gas compressor station that can overcome the shortcomings mentioned above and does not require a direct monitoring by an operator or can be remotely monitored by a central dispatcher.

\section{Industrial automation systems}

In many cases, the unfolding of a production process requires physical parameters such as pressure, flow or temperature to either be maintained at a constant level or to vary over time according to specific laws.

In practice, this can be achieved by using specialised automation equipments called automated regulators that maintain the parameter at the desired level in an automated manner, without human intervention [5].

Such regulators can be used for controlling parameters as diverse as the feed rate and precision for a contouring equipment $[6,7]$ or the pressure in a natural gas transportation or compression system.

A regulator can work either in manual mode or in automated mode.

A first step in starting a regulation process is the tuning of the regulator, by successively modifying the work parameters so that the system reacts according to the requirements of the process [5].

Function of the type of dependence between the control parameter and the error, automated regulation systems can be proportional (P), proportional-integrative (PI) or proportional-integrative-derivate (PID). 
Figure 1 shows the standard configuration of an automated regulation and control system [5].

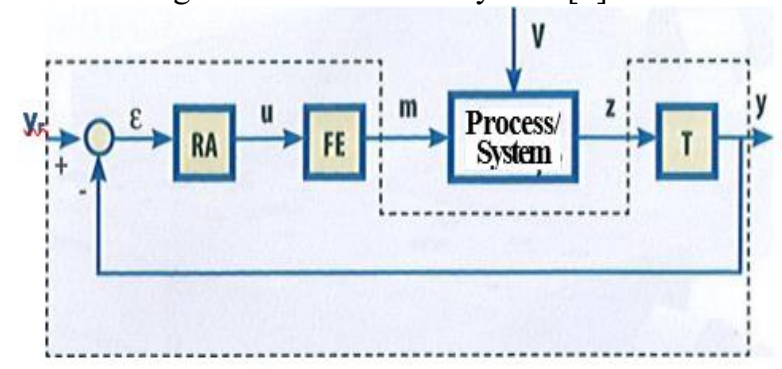

Fig. 1. The basic structure of an automated regulation and control system [5]

$\mathrm{RA}=$ automated regulator; $\mathrm{EE}=$ execution element; $\mathrm{T}=$ transducer

$\mathrm{y}_{\mathrm{r}}=$ reference value; $\mathrm{y}=$ output value; $\varepsilon=$ error; $\mathrm{u}=$ control parameter; $\mathrm{m}=$ execution parameter; $\mathrm{v}=$ disturbance; $\mathrm{z}=$ quality

The most common type in use today is the PID regulator. The control parameter can be determined with a function of the type [5]:

$u(t)=K_{R}^{*} \varepsilon(t)+K_{i}^{*} \int \varepsilon(t) d t+K_{D} *_{\varepsilon}(t) / d t$

PID regulators are employed especially when there are larger disturbances in the system. The $\mathrm{P}$ component reduces the overregulation and leads to a short transition time, but also introduces a large stationary error. The I component cancels the stationary error but introduces a larger overregulation and a longer response time. In turn, the $\mathrm{D}$ component improves the dynamic behaviour, introduces small values of the overregulation and of the transition time but introduces a large stationary error. Their combination therefore leads to better performance both in terms of the overregulation, of the transition time and of the size of the stationary error $[1,5,6]$.

Figure 2 [5] shows the response of a PID automated regulator to a step-type input.

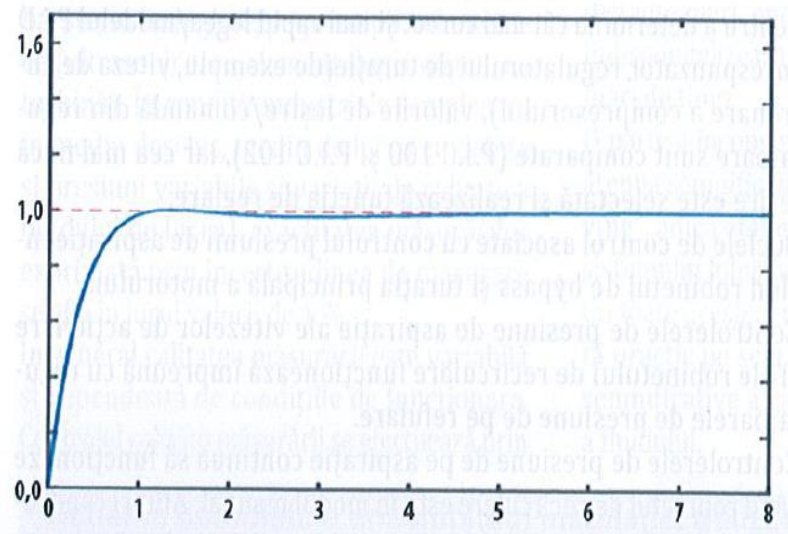

Fig. 2. Response of a PID regulator to a step-type input.

\section{The new automation system implemented in the natural gas compressor station}

Given the fact that the existing equipment within the natural gas compressor station does not allow for the automated regulation of the gas pressure, it was considered as necessary to implement a new automation solution to solve this problem.

One of the main problems that needed to be solved was which component to use as execution element for the automation (control) system, or more precisely on what type of valve to fit it. The compressor systems comprise two main types of valves - slide valves and spherical shutter valves. However, the automation system cannot be fitted directly on a spherical shutter valve, because this type of valve is not designed to work in an intermediate position, as this would subject the valve's sphere to intensive corrosion, which in turn would lead to a deteriorated surface and to leaks. Therefore, the automation system is fitted on a slide valve that is better suited for this type of operation.

The main elements of the implemented automation system are as follows:

- a BIFFI ICON 2000 electric actuator fitted on the slide valve (figure 3 ) and a programmable automation and monitoring panel that will be installed in the control room of the natural gas compressor station;

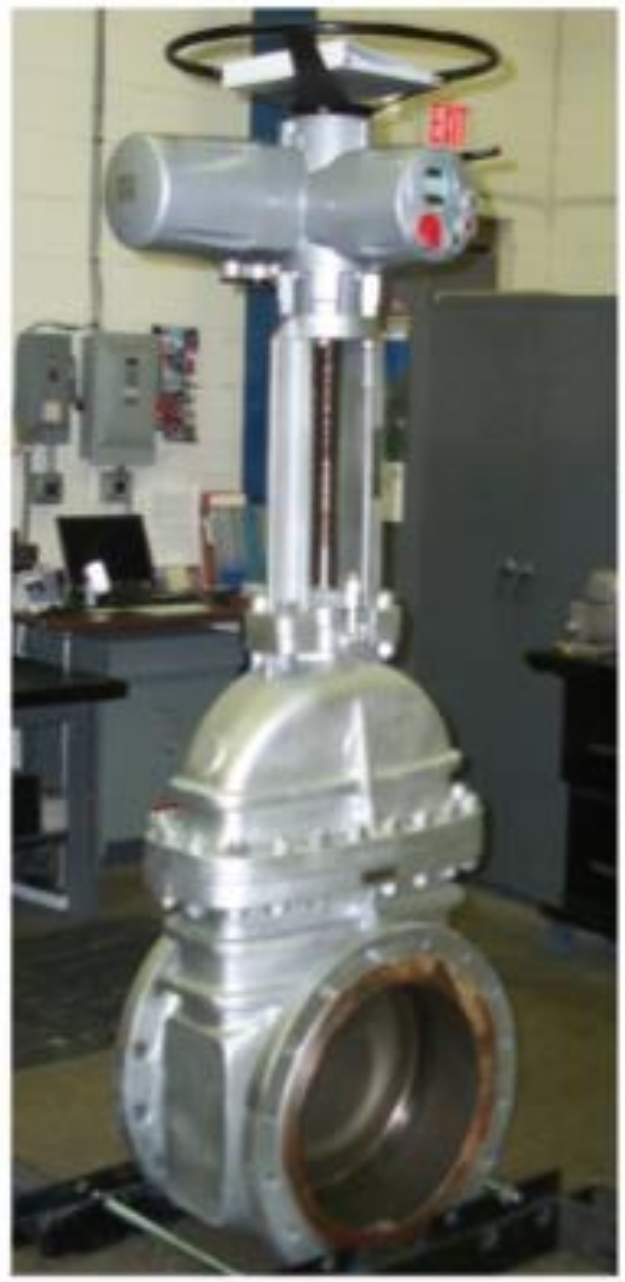

Fig. 3. BIFFI ICON 2000 electric actuator fitted onto a disassembled valve.

a process computer equipped with a power source, input-output and communication modules and with clips for electrical connections;

- $\quad$ HMI (human machine interface) operation panel with an adequate display. 
- automatic circuit breaker for overload and shortcircuit protection for the general power circuit and for the power circuit of the controlled valve.

Also, in order to provide for the automated regulation of the gas pressure, it is necessary to install some pressure transducers, each of which will transmit to the automated regulation system a signal of 4-20 mA, proportional to the value of the pressure in the measuring point:

- a pressure transducer for the aspiration part of the compressor, installed ahead of the regulation valve, with a measurement range of $0-60 \mathrm{bar}-\mathrm{PT} 1$;

- a pressure transducer for the aspiration part of the compressor, installed below the regulation valve, with a measurement range of $0-60 \mathrm{bar}-\mathrm{PT} 2$;

- a pressure transducer for the exhaust part of the compressor, with a measurement range of 0-100 bar-PT3.

All three transducers will provide the regulation system (the automation panel) with the realtime values of the three pressures, values that represent the input for the calculation of the control parameter of the regulation system, parameter that will be also sent through an unified 4-20 mA current signal to the electrical actuator BIFFI ICON2000 (figure 4 [8]), fitted on the slide valve.

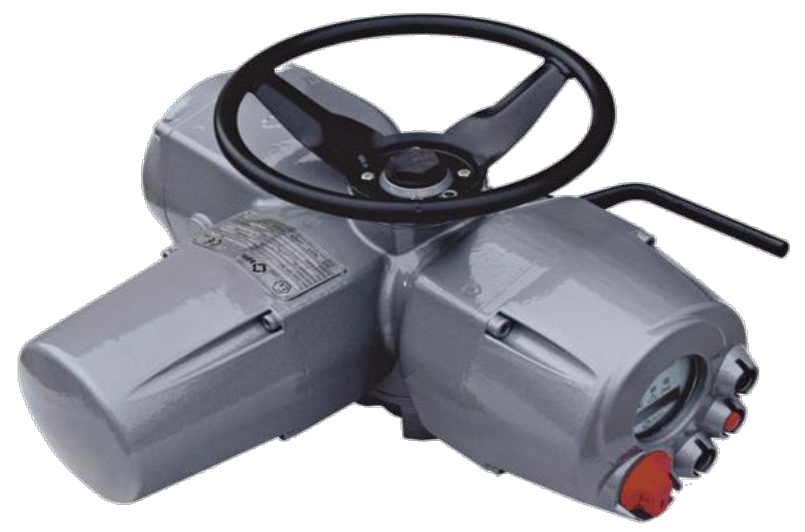

Fig. 4. Electric actuator BIFFI ICON 2000 [8]

The electric actuator BIFFI ICON 2000 has following characteristics [6]:

- Power supply: either 3 phase from $208 \mathrm{~V}$ to $690 \mathrm{~V}$ at $50 / 60 \mathrm{~Hz}$ or 1 phase from $110 \mathrm{~V}$ to $240 \mathrm{~V}$ at $50 / 60 \mathrm{~Hz}$

- Torque output: From 30 to 57,000 Nm

- Speed range: From 12 to $173 \mathrm{RPM}$ at $50 / 60 \mathrm{~Hz}$

- Work temperature range: $-20^{\circ} \mathrm{C}$ to $+85^{\circ} \mathrm{C}$

- Non-intrusive configuration;

- User-friendly push-button panel for operation, setting and diagnostics;

- Bluetooth ${ }^{\mathrm{TM}}$ wireless connectivity;

- Advanced maintenance data and alarm reports;

- Valve condition monitoring;

- Configurable 'data logger' function for maintenance and diagnostic programs in recorder or event modes;

- Customized numeric and graphic displays with 8 language options;

- Single enhanced terminal block;

- Digital contactless torque and position sensing;

- Watertight and explosionproof PDAs available;

- $\quad$ Suitable for use in SIL 2 applications.
Also, the automation panel will be connected to the spherical shutter valve, that can be controlled manually from the HMI part of the automation panel, should this become necessary during the compression process.

Each of the two valves can be controlled manually only after introducing a user-specific password and any of these manoeuvres will be automatically stored and included in a report that can be visualised later on the HMI display.

Also, all essential information from the regulation process can be remotely visualised by means of mobile devices connected through GPRS to the process controller.

In order to ensure the system's cybernetic security there will be used a GSM data card with fixed IP and the access to the information provided by the controlled will require the usage of user names and of the corresponding passwords. It will not be possible to issue a command for the modification of the valves' position by GPRS communication, since this connection will be dedicated exclusively to the reading of data from the regulated process. The only possibility for controlling the valves or for cancelling the controlled pressure will be by means of the HMI installed on the frontal panel of the automation system or by using the buttons of the local panels for the operation of the electric actuators BIFFI ICON 2000 fitted on the slide valve and on the spherical shutter valve.

In turn, the controller will communicate with a remote process computer by GPRS for the transfer of acquired data and for acquiring the targeted values of the regulated process variables. In order to visualise the parameters, the prescription of the regulated process variables and the control of the execution elements connected to the controller, independently from the process computer, there will be used a 15 " touchscreen installed on the front panel of the automation block. This panel will allow the operation of the two valves, the monitoring of the regulation process from the compressors' aspiration part, as well as the visualisation of data on mobile devices by means of GPRS connections.

As can be seen in figure 5, the HMI display allows the visualisation of the three pressure values in both analogical and digital form, as well as the position of the valve $(100 \%$ open). The visualisation of the functioning parameters and of the valves' position allows a parametrisation of the control function. By means of the HMI, the operator can supervise all process parameters and can compare the values of the various displayed parameters, so that he can then make quick decisions function of the indicated pressure value.

The situation presented in figure 5 in terms of the displayed values requires some further discussion. The value of the set pressure is higher than that of the pressure registered ahead of the control valve (PT1). In this situation, it can be assumed that the controller will be unable to bring the pressure to the targeted value, as even in the case in which the valve is $100 \%$ open, the pressure ahead of the valve can be at most equal to that behind the valve. This is a particular case for which the control system has to be programmed in such a manner that it alerts the operator if the value is too high. 


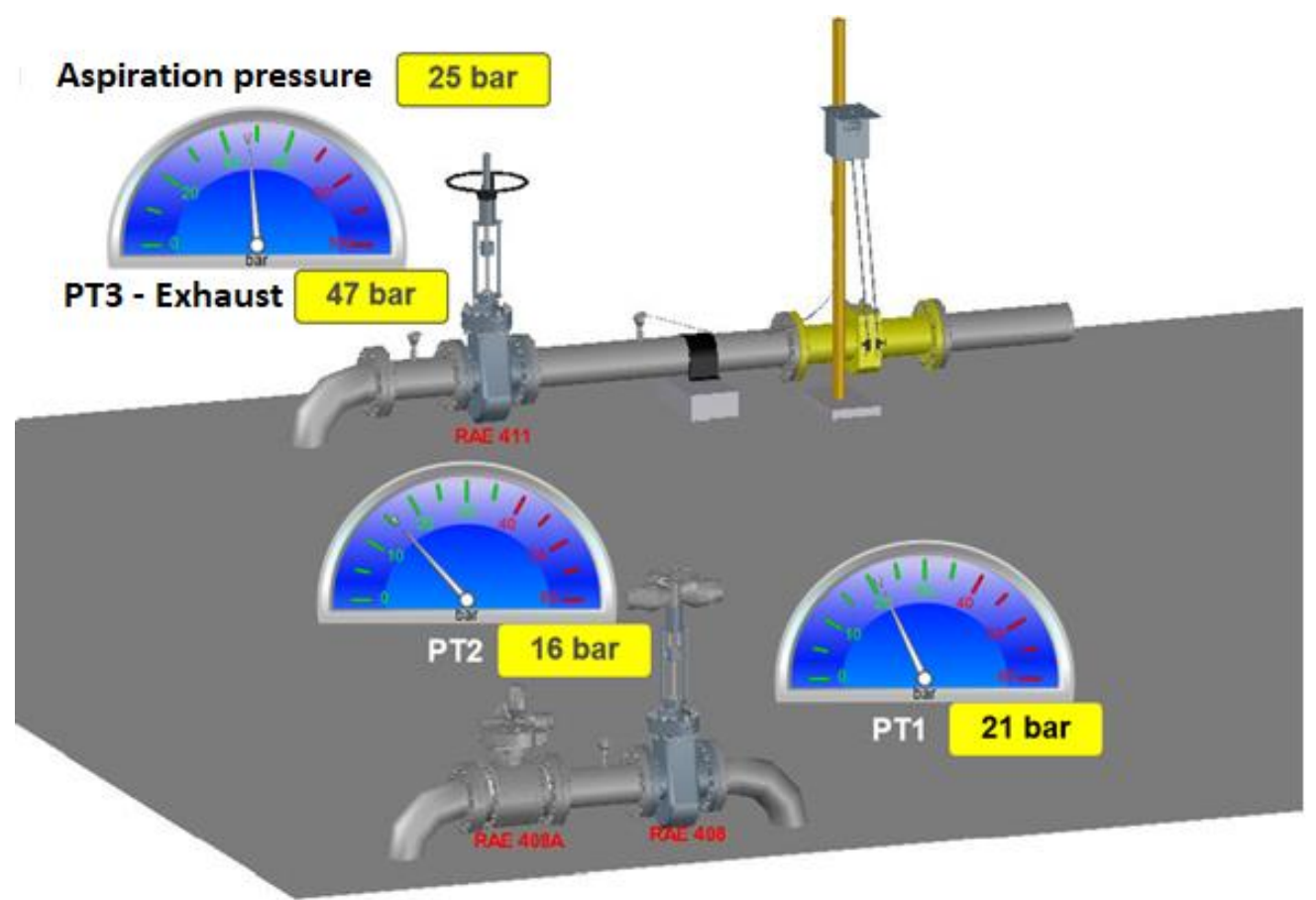

Fig. 5. The graphical interface developed on the HMI of the implemented automation system, showing the valves, the transducers and the controlled parameters.

\section{Conclusions}

The paper has described the implementation of an automation system for pressure control in a natural gas compressor station.

By implementing such a control system, the compressor station can work more efficiently and the gas injection process will be much easier to monitor, since the operator will have quick access to all information and can make informed decisions. Furthermore, this information can get directly to the decision factors in the company by means of the process computer that communicates with the controller.

All data are stored in the process computer and there can be made real time analyses of the functioning of the control system and of the process of injecting natural gas into a gas storage facility.

The new control system therefore represents a step towards achieving a higher efficiency and lower exploitation costs for natural gas compressor stations.

In future, the author will expand her researches in order to improve this automation solution and to apply improved automation solutions also to other elements within the natural gas life cycle.

\section{References}

1. I. Foidas, Testing and production of natural gas deposits (Translation from Romanian) (Publishing House of the „Lucian Blaga” University of Sibiu, 2014)

2. A.-S. Huidan, Electrical equipment for automation and control in environments with explosion hazard (Translation from Romanian) (Technical Publishing House, 2008)

3. L. Tarnu, Elements of Law and Legislation (Translation from Romanian) (Publishing House of the „Lucian Blaga” University of Sibiu, 2014)

4. L. Tarnu, International protection of human rights (Translation from Romanian) (Tehnomedia Publishing House, 2014)

5. O. Tanase, Rev. Automatiz. Instrum., 27, 1 (2018)

6. R. Breaz, O. Bologa, M. Tera, C. Deac, Proc. 9th IEEE Intl. Conf. Ind. Inf., 1, (2011)

7. C. Biriș, C. Deac, M. Tera, Annals DAAAM, 1 (2008)

8. BIFFI, ICON 2000 Electric actuators, https://biffi.it/en-us/biffi-products/biffi-electricactuators/biffi-icon-2000, 2018 